\title{
Extravasated platelet aggregation in liver zone 3 may correlate with the progression of sinusoidal obstruction syndrome following living donor liver transplantation: A case report
}

\author{
SHINICHI NAKANUMA, TOMOHARU MIYASHITA, HIRONORI HAYASHI, HIDEHIRO TAJIMA, \\ HIROYUKI TAKAMURA, TOMOYA TSUKADA, KOICHI OKAMOTO, SEISHO SAKAI, ISAMU MAKINO, \\ JUN KINOSHITA, KEISHI NAKAMURA, KATSUNOBU OYAMA, MASAFUMI INOKUCHI, \\ HISATOSHI NAKAGAWARA, ITASU NINOMIYA, HIROHISA KITAGAWA, \\ SACHIO FUSHIDA, TAKASHI FUJIMURA and TETSUO OHTA
}

Department of Gastroenterologic Surgery, Division of Cancer Medicine, Graduate School of Medical Science, Kanazawa University, Kanazawa, Ishikawa 920-8641, Japan

Received April 19, 2014; Accepted January 5, 2015

DOI: $10.3892 / \mathrm{etm} .2015 .2245$

\begin{abstract}
Sinusoidal obstruction syndrome (SOS), previously known as veno-occlusive disease, is relatively rare subsequent to liver transplantation (LT). SOS refractory to medical therapy, however, can result in centrilobular fibrosis, portal hypertension and liver failure. Although sinusoidal endothelial cell damage around central venules (zone 3 ) occurs early in the development of SOS, the detailed mechanism of SOS development and its association with thrombocytopenia are not yet completely understood. The present report describes a patient who experienced SOS with unexplained thrombocytopenia following living donor LT. The progression of SOS resulted in graft dysfunction and the patient succumbed. The presence of platelets in the liver allograft was assayed immunohistochemically using antibody to the platelet marker cluster of differentiation $42 \mathrm{~b}$ (platelet glycoprotein Ib). Platelet aggregates were found attached to hepatocytes along the sinusoid and within the cytoplasm of hepatocytes, particularly in zone 3 . By contrast, no staining was observed in zone 1 . These findings suggested that extravasated platelet aggregation in the space of Disse and the phagocytosis of platelets by hepatocytes were initiated by sinusoidal endothelial cell damage due to the toxicity of the immunosuppressant tacrolimus or a corticosteroid pulse, and that platelet activation and degranulation may be at least partially involved in the mechanism responsible for SOS.
\end{abstract}

Correspondence to: Dr Shinichi Nakanuma, Department of Gastroenterologic Surgery, Division of Cancer Medicine, Graduate School of Medical Science, Kanazawa University, 13-1 Takaramachi, Kanazawa, Ishikawa 920-8641, Japan

E-mail: n_shin@gj8.so-net.ne.jp

Key words: platelet, aggregation, cluster of differentiation $42 \mathrm{~b}$, sinusoidal obstruction syndrome, veno-occlusive disease, liver transplantation

\section{Introduction}

Sinusoidal obstruction syndrome (SOS), a condition previously known as veno-occlusive disease (1), is a life-threatening syndrome that results from sinusoidal congestion and is characterized by hepatomegaly, ascites, portal hypertension, weight gain and jaundice (2). This syndrome is commonly observed as a complication of high-dose chemotherapy administered prior to hematopoietic progenitor cell (HPC) transplantation (3). The development of SOS subsequent to liver transplantation (LT) is relatively uncommon, being observed in $\sim 2 \%$ of LT recipients (4). SOS is severe and refractory to medical therapy, with the ultimate solution being re-transplantation (2).

The mechanism underlying the development of SOS is not yet completely understood, although sinusoidal endothelial cell damage leading to alterations in the hemostatic system is regarded as central to the pathogenesis of SOS (5). SOS has additionally been reported to be associated with platelet functions. For example, transforming growth factor (TGF) $\beta-1$ secreted by platelets has been shown to contribute to hypercoagulability in SOS following HPC transplantation (6). In addition, plasminogen activator inhibitor-1 (PAI-1), which is abundant in platelets, has been suggested to be a specific marker of SOS (7). Clinically, patients who experience SOS following HPC transplantation are both thrombocytopenic and refractory to platelet transfusions (8). These observations suggest that platelets may be important in the pathogenesis of SOS.

The present case report describes a patient who experienced liver allograft dysfunction caused by the progression of SOS following living donor LT (LDLT), with unexplained thrombocytopenia occurring around the same period of time. We hypothesized that the platelets had been consumed by the liver allograft, and therefore the allograft was immunohistochemically assayed for the presence of platelets using antibody to the platelet marker cluster of differentiation (CD) $42 b$ (platelet glycoprotein Ib). 


\section{Case report}

A 59-year-old male patient was diagnosed with primary biliary cirrhosis-autoimmune hepatitis overlap syndrome and end-stage cirrhosis. His Child-Pugh score was $\mathrm{C} 12$ points and his Model For End-Stage Liver Disease score was 23 (9). The donor was the patient's 56-year-old wife. Their blood types were compatible (blood type $\mathrm{O} \rightarrow \mathrm{AB}$ ), and the lymphocyte cross-match test was negative. The LDLT involved the right lobe without the middle hepatic vein $(500 \mathrm{~g})$. The graft-to-recipient weight ratio was 0.83 , with the graft constituting $42 \%$ of the standard liver volume of the recipient. Following transplantation, the patient was started on immunosuppressive treatment with tacrolimus (Tac), mycophenolate mofetil (MMF) and prednisolone. His immediate postoperative course was good, and he was moved from the intensive-care unit on postoperative day (POD) 3.

Approximately 2 months after the LDLT, the patient's concentrations of biliary tract enzymes and total bilirubin (T-Bil) began to increase progressively. Endoscopic retrograde cholangiopancreatography revealed no stenosis of the biliary anastomosis, and color doppler ultrasonography and computed tomography revealed no abnormalities. A liver biopsy on POD 77 showed minimal inflammation of the portal area and slight endothelial inflammation of the central venules. At that time, the patient's platelet counts were $<10 \times 10^{5} / \mathrm{mm}^{3}$ and his T-Bil concentration continued to increase, to $17 \mathrm{mg} / \mathrm{dl}$. The patient was regarded as experiencing late-onset acute rejection and was treated with augmented immunosuppressive therapy, consisting of intravenous corticosteroid pulse therapy, increased doses of MMF and adjustment of the Tac trough level to maintain a whole-blood concentration of 5-10 ng/ml. A liver biopsy taken on POD 91 showed minimal inflammation and no bile duct injury in the portal area (Fig. 1A), similar to the previous biopsy. Zone 3, however, showed chronic inflammation and a loss of hepatocytes (Fig. 1B). The sample was negative for $\mathrm{C} 4 \mathrm{~d}$ staining, a marker of antibody-mediated rejection. Based on these findings, the patient was diagnosed with late-onset acute rejection accompanied by mild central perivenulitis, resulting in an intensification of immunosuppression. Since the concentration of Tac could not be maintained $>10 \mathrm{ng} / \mathrm{ml}$, despite increased doses, the patient's calcineurin inhibitor treatment was switched from Tac to cyclosporin A (CsA), with the CsA dose adjusted to maintain a target level in the blood of $150-200 \mathrm{ng} / \mathrm{ml}$, and everolimus was added as a third immunosuppressive agent. In addition, the patient was administered two doses of basiliximab (50 $\mathrm{mg} /$ day) to suppress the antibody-mediated rejection. The patient's platelet counts declined to $\sim 5 \times 10^{5} / \mathrm{mm}^{3}$, despite the lack of any apparent causes of the thrombocytopenia, such as disseminated intravascular coagulation, thrombotic microangiopathy or any other thrombotic diseases; however, his T-Bil concentration increased to $21.4 \mathrm{mg} / \mathrm{dl}$. A liver biopsy taken on POD 203 showed inflammatory cells infiltrating the liver parenchyma in zone 3 , as well as marked reductions in hepatocytes and central vein obliteration, as revealed by hematoxylin and eosin staining (Fig. 1C). Masson's trichrome stain showed basement membrane formation caused by fibrous tissue and sinusoidal fibrosis (Fig. 1D). By contrast, the portal area (zone 1) showed minimal inflammation, similar to the previous biopsy. The patient's central perivenulitis had progressively worsened, resulting in the development of SOS. His platelet count was $\sim 5 \times 10^{5} / \mathrm{mm}^{3}$, the allograft was less responsive to augmented immunosuppressive therapy and his condition was considered irreversible. Re-transplantation was indicated if feasible, but a donor could not be found. He subsequently experienced liver and renal failure and succumbed on POD 250.

Immunohistochemistry of $C D 42 b$. The presence of platelets in the liver tissue samples was assayed using a mouse monoclonal antibody to CD42b (1:100; cat. no. EPR6995; Abcam, Tokyo, Japan), a marker expressed on both inactive and activated platelets. In normal spleen tissue, used as a positive control, CD42b expression was evident as dots, morphologically characterized as platelets (Fig. 2A), whereas there was no staining of normal liver tissue, used as the negative control (Fig. 2B). Assay of the liver allograft tissue obtained from the patient on POD 91 showed CD42b expression in zone 3 (Fig. 3A). At greater magnification, the $\mathrm{CD} 42 \mathrm{~b}$ appeared as aggregates attached to hepatocytes along the sinusoids. CD42b was also expressed in the cytoplasm of the hepatocytes (Fig. 3B) but was not observed in zone 1 .

\section{Discussion}

SOS is believed to result from sinusoidal endothelial cell damage in zone 3 of the liver (5). Red blood cells subsequently leak into the space of Disse, depositing fibrin (1). The basement membrane that forms from the fibrous tissue leads to hepatocyte ischemia, followed by hepatocyte necrosis (1). Tac administration following transplantation may be due to sinusoidal endothelial cell damage of the small hepatic vein in zone $3(10,11)$. To date, however, the pathophysiology of SOS remains incompletely understood, with no definitive mechanisms or effective treatments identified.

There are few reports concerning a pathologic association between platelets and SOS. One study, in which liver samples taken at autopsy from HPC transplant recipients with SOS were stained with an anti-platelet antibody, found no evidence of platelet deposition (12); however, to prevent SOS, its pathogenetic mechanism must be clarified prior to it becoming irreversible. The present study therefore assessed liver tissue samples taken from an LDLT recipient in the development process of SOS. Zone 3 of the liver was positive for CD42b, with the staining pattern observed as aggregates attached to hepatocytes along the sinusoid and in the cytoplasm of the hepatocytes. These findings suggest that extravasated platelet aggregation (EPA) was present in the space of Disse, and that the platelets were phagocytized by hepatocytes. It is likely that these aggregated and activated platelets in the space of Disse had a greater influence on hepatocytes than did the platelets in the sinusoidal vessels.

Liver sinusoids are a type of sinusoidal blood vessel with endothelial fenestrations (13) (Fig. 4A), the diameters of which are increased by sinusoidal endothelial cell damage (14). The sinusoidal endothelial cells in the patient described in the present report had been exposed to Tac or a corticosteroid pulse, resulting in sinusoidal endothelial cell damage. This damage likely contributed to the denuding of the endothelium or loss of the fenestrations, allowing platelets to enter the space of Disse (Fig. 4B). This space contains reticulin fibers, most of which contain collagen type 3 (15). Platelets have been found to bind 

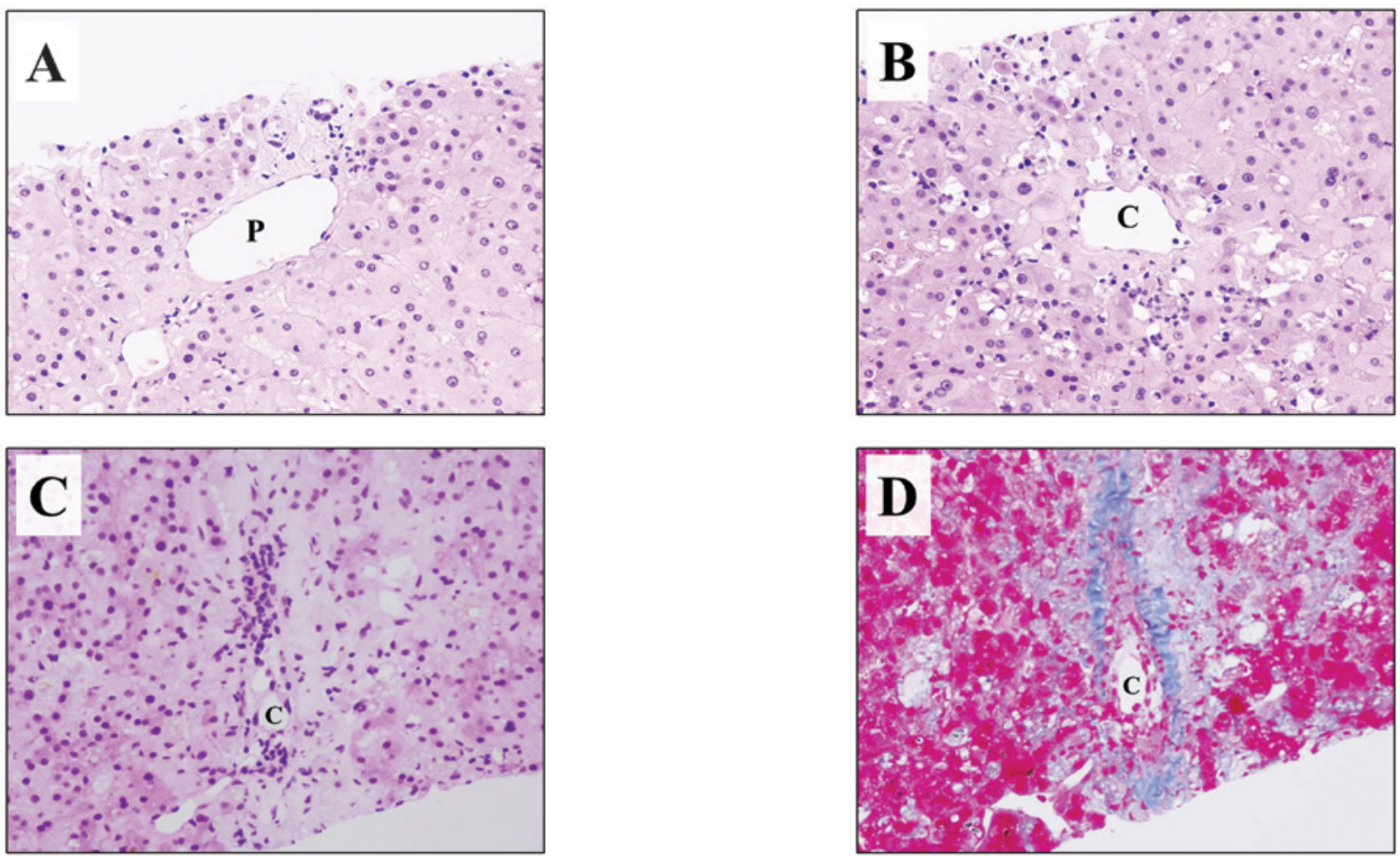

Figure 1. Histopathology of the liver biopsy sample obtained on POD 91 and 203 (magnification, x200). (A and B) Samples taken on POD 91 showing (A) minimal inflammation and no bile duct injury in the portal area and (B) chronic inflammation and loss of hepatocytes in zone 3 (hematoxylin and eosin staining) (C and D) Samples taken on POD 203 showing (C) inflammatory cells infiltrating into the liver parenchyma, severe loss of hepatocytes and central vein obliteration (hematoxylin and eosion staining) and (D) basement membrane formation caused by fibrous tissue and sinusoidal fibrosis, with progressive worsening of the central perivenulitis and the development of sinusoidal obstruction syndrome (Masson's trichrome stain). C, central vein; $\mathrm{P}$, portal vein; POD, postoperative day.
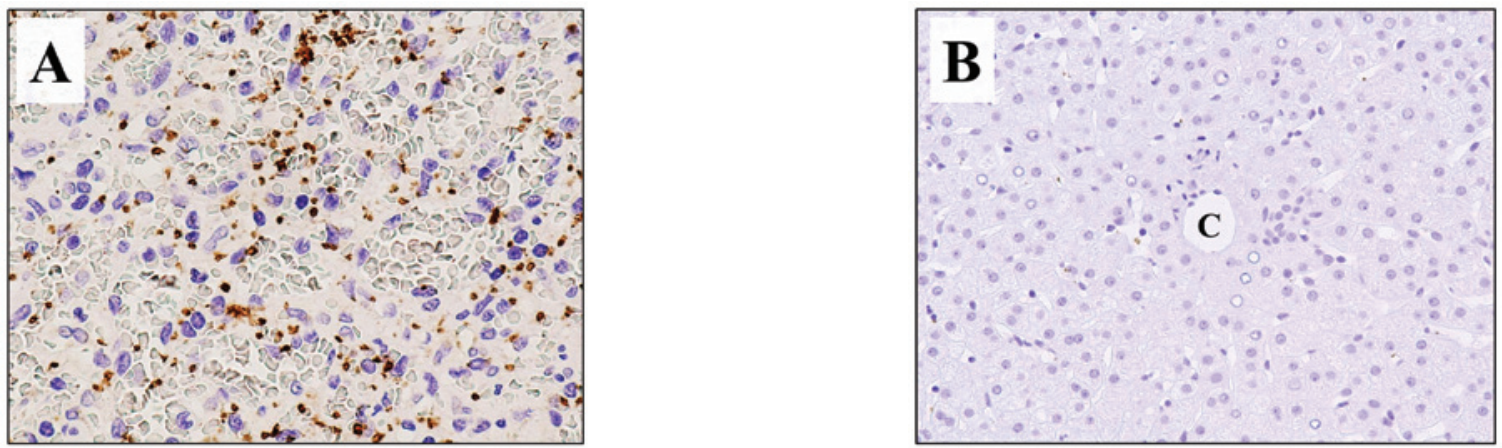

Figure 2. Immunohistochemical staining of (A) normal spleen and (B) normal liver with antibody to cluster of differentiation 42b. (A) Immunoreactivity was evident throughout the spleen as dots, which were morphologically characterized as platelets (magnification, $\mathrm{x} 400$ ). (B) No staining of normal liver tissue was observed (magnification, x200). C, central vein.
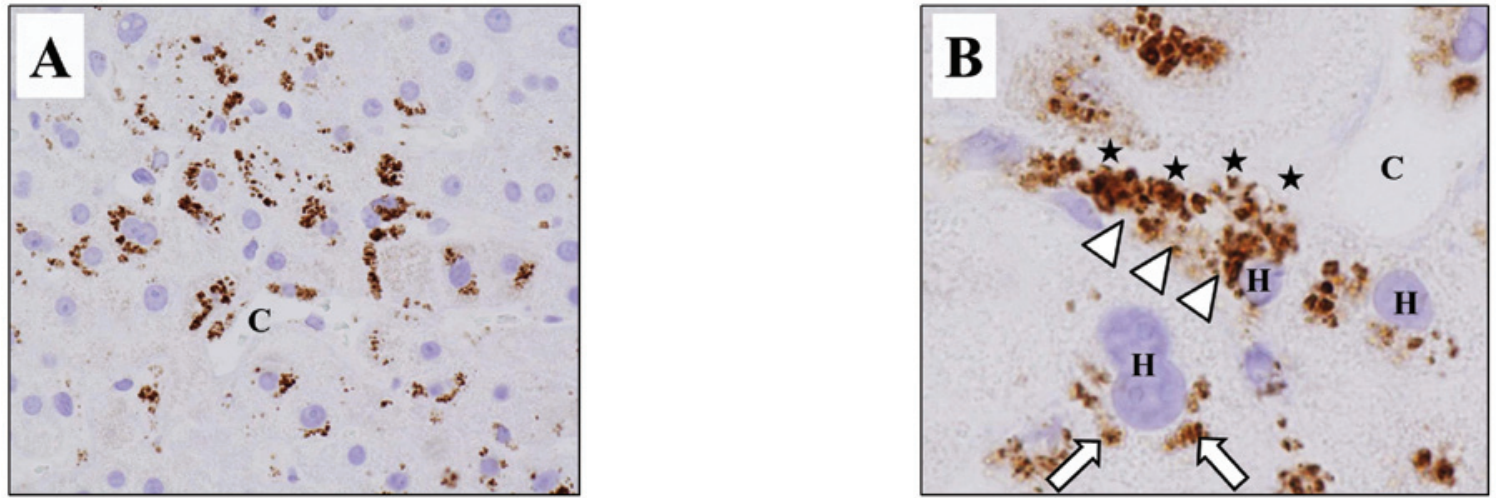

Figure 3. Immunohistochemical staining of liver allograft tissues with antibody to CD42b. (A) The liver biopsy taken on postoperative day 91 showed CD42b expression in zone 3 (magnification, $\mathrm{x} 400$ ). (B) At higher magnification, CD42b was present as aggregates attached to hepatocytes (arrowheads) along the sinusoid ( $\star$ ) and in the hepatocyte cytoplasm (arrows) (magnification, x1,000). C, central vein; H, hepatocyte; CD42b, cluster of differentiation 42b. 

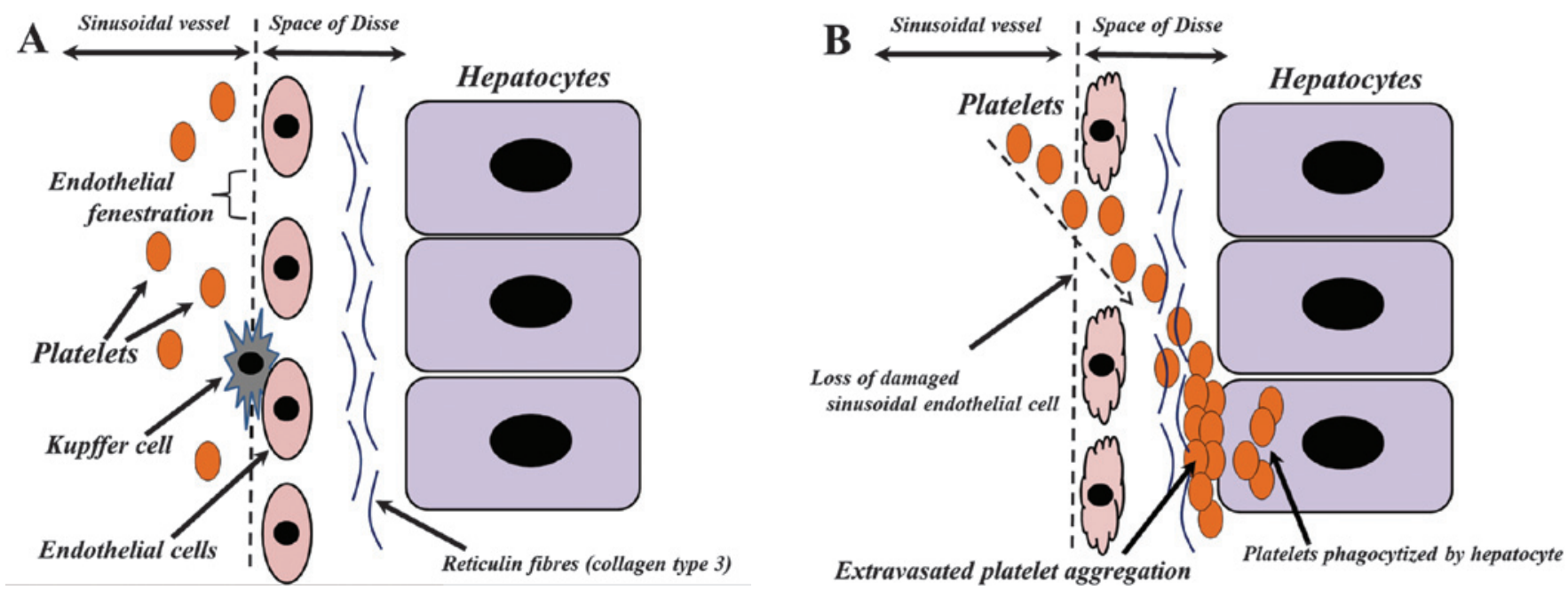

Figure 4. Schematic model of (A) a normal sinusoid and (B) the sinusoid of sinusoidal obstruction syndrome in the patient in the present study. The normal sinusoid is a type of sinusoidal blood vessel with endothelial fenestrations (A). Damage to the sinusoidal endothelium can result in the denuding of the endothelium or the loss of fenestrations, allowing platelets to enter the space of Disse. This space contains reticulin fibers, which consist primarily of collagen type 3. Platelets can easily attach to collagen type 3, forming aggregates, including in the space of Disse. In addition, the extravasated platelets in the space of Disse can be phagocytized by hepatocytes through the asialoglycoprotein receptor (B).

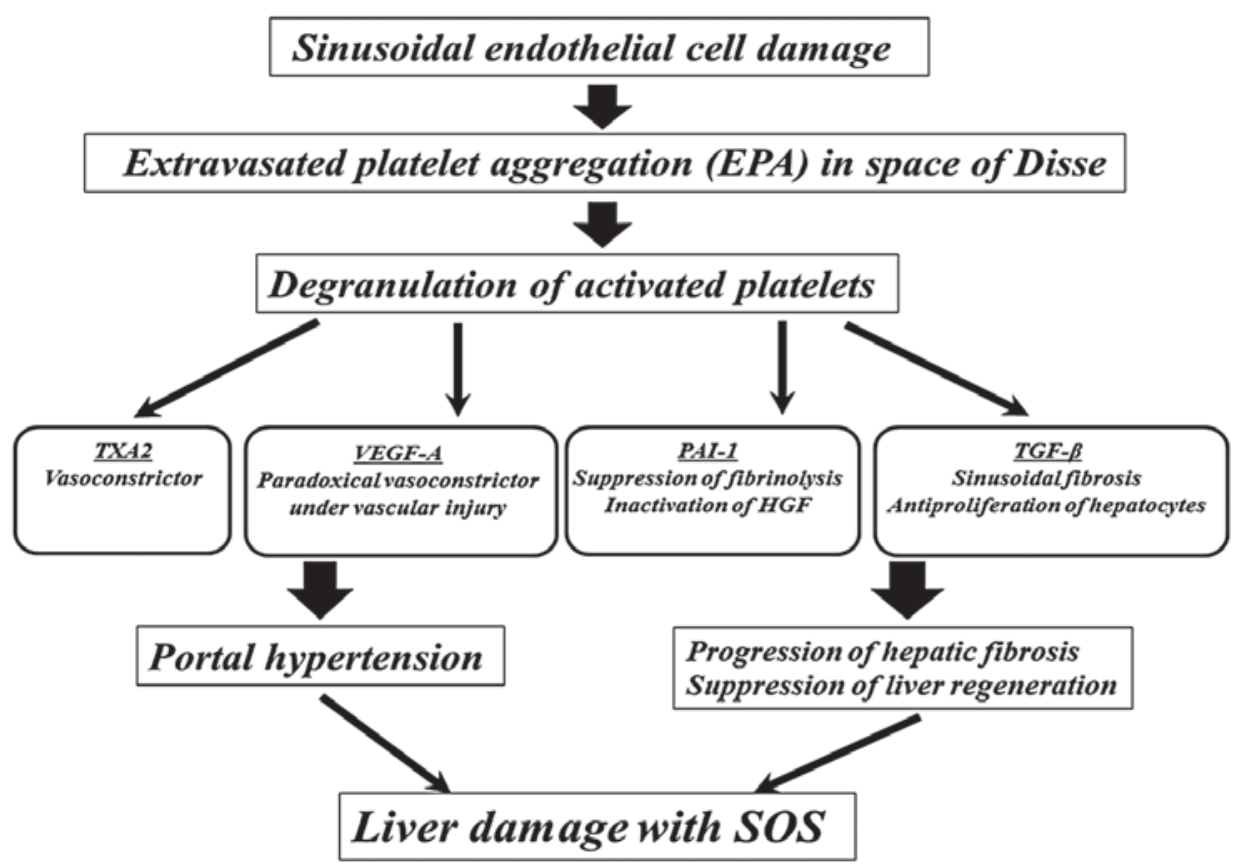

Figure 5. The predicted pathogenic mechanism of SOS in the patient in the present study. Extravasated platelet aggregation in the space of Disse was initiated by damage to the sinusoidal endothelium induced by tacrolimus or a corticosteroid pulse. The negative regulators released by activated platelets, including TXA2, VEGF-A, PAI-1 and TGF- $\beta$, may have induced portal hypertension and the progression of hepatic fibrosis, as well as suppressed liver regeneration, initiating liver damage with SOS. SOS, sinusoidal obstruction syndrome; TXA2, thromboxane A2; VEGF-A, vascular endothelial growth factor-A; PAI-1, plasminogen activator inhibitor-1; HGF, hepatocyte growth factor; TGF- $\beta$, transforming growth factor- $\beta$.

to and form aggregates with collagen type 3 (16), resulting in platelet aggregation in the space of Disse (Fig. 4B).

Hepatocytes contribute to blood homeostasis by endocytosing a number of proteins present in plasma (17). The asialoglycoprotein receptor (ASGR), a membrane-bound lectin, removes the target glycoproteins from the circulation (18). These glycoproteins are also found on the surface of platelets, and human platelets have been reported to be sequestered by hepatocytes through the ASGR (19). In the patient in the present report, the extravasated platelets in the space of
Disse may have been phagocytized by hepatocytes through the ASGR (Fig. 4B).

Platelets contain $\alpha$ and dense granules (20). Upon activation, platelets excrete the contents of these granules into their canalicular systems or the surrounding blood (20). These granules also contain negative regulators of liver regeneration, including thromboxane A2 (TXA2), vascular endothelial growth factor-A (VEGF-A), TGF- $\beta$ and PAI-1 $(6,21,22)$. TXA2 is a vasoconstrictor that increases portal venous resistance (23) and causes portal hypertension. Although VEGF-A acts as 
a vasodilator under ordinary circumstances, it acts, paradoxically, as a vasoconstrictor in patients with endothelial failure (24). Bevacizumab, an antibody against VEGF-A, protects against liver injury associated with SOS (25). PAI-1 suppresses fibrinolysis and the progression to fibrosis in the tissue microenvironment. In addition, PAI-1 acts as a negative regulator of hepatocyte proliferation by inhibiting urokinase-type plasminogen activator, which activates hepatocyte growth factor $(26,27)$. TGF- $\beta$ is a major antiproliferative factor for hepatocytes (28) that stimulates collagen synthesis through activated hepatic stellate cells (29). In the patient in the present report, the release by platelets of these negative regulators may have induced portal hypertension and the progression of hepatic fibrosis, as well as the suppression liver regeneration, a mechanism responsible, at least in part, for the initiation of liver damage with SOS (Fig. 5).

No standard method of treating SOS has yet been established. Systemic anticoagulation and thrombolytic therapies have been tested extensively (30). Defibrotide, a polydeoxyribonucleic acid, has been recently shown to have a promising response rate in patients with severe SOS (31). When medical treatment fails, transjugular intrahepatic portosystemic shunt placement or re-transplantation can be considered (2). Depending on the extent of the sinusoidal endothelium damage and EPA in the space of Disse, the prophylactic administration of endothelial protective and antiplatelet agents may be effective prior to the development of irreversible damage. Cilostazol, a phosphodiesterase 3 (PDE3) inhibitor, may be appropriate, owing to its antiplatelet properties, its ability to increase tolerance to ischemia/reperfusion injury (32) and its induction of immune tolerance via an enhanced regulatory T-cell response (33). In clinical practice, we have administered a PDE3 inhibitor to LDLT recipients, with favorable outcomes (Nakanuma et al, unpublished data).

In conclusion, the present findings suggest that EPA in the space of Disse, which was initiated by sinusoidal endothelial cell damage due to the toxicity of Tac or a corticosteroid pulse and the negative regulators released by activated platelets, may have partially contributed to liver damage with SOS in the patient. Endothelial protective therapy or antiplatelet treatment may have been useful in the treatment of SOS in this patient. Studies in additional patients are necessary to elucidate the role of EPA in the space of Disse on the development of SOS.

\section{References}

1. Kitajima K, Vaillant JC, Charlotte F, et al: Intractable ascites without mechanical vascular obstruction after orthotopic liver transplantation: etiology and clinical outcome of sinusoidal obstruction syndrome. Clin Transplant 24: 139-148, 2010.

2. Campos-Varela I, Castells L, Dopazo C, et al: Transjugular intrahepatic portosystemic shunt for the treatment of sinusoida obstruction syndrome in a liver transplant recipient and review of the literature. Liver Transpl 18: 201-205, 2012.

3. Membreno FE, Ortiz J, Foster PF, Wright F, et al: Liver transplantation for sinusoidal obstructive syndrome (veno-occlusive disease): case report with review of the literature and the UNOS database. Clin Transplant 22: 397-404, 2008.

4. Sebagh M, Debette M, Samuel D, et al: 'Silent' presentation of veno-occlusive disease after liver transplantation as part of the process of cellular rejection with endothelial predilection. Hepatology 30: 1144-1150, 1999.

5. Coppell JA, Brown SA and Perry DJ: Veno-occlusive disease: cytokines, genetics, and haemostasis. Blood Rev 17: 63-70, 2003.
6. Pihusch V, Pihusch M, Penovici M, et al: Transforming growth factor beta-1 released from platelets contributes to hypercoagulability in veno-occlusive disease following hematopoetic stem cell transplantation. Thromb Res 116: 233-240, 2005.

7. Salat C, Holler E, Kolb HJ, et al: Plasminogen activator inhibitor-1 confirms the diagnosis of hepatic veno-occlusive disease in patients with hyperbilirubinemia after bone marrow transplantation. Blood 89: 2184-2188, 1997.

8. Oh H, Tahara T, Bouvier M, et al: Plasma thrombopoietin levels in marrow transplant patients with veno-occlusive disease of the liver. Bone Marrow Transplant 22: 675-679, 1998.

9. Wiesner RH, McDiarmid SV, Kamath PS, et al: MELD and PELD: application of survival models to liver allocation. Liver Transpl 7: 567-580, 2001

10. Chen H, Wang X, Fan T, et al: A case of veno-occlusive disease following liver transplantation. Exp Ther Med 7: 141-144, 2014.

11. Wang SE, Shyr YM and Lee RC: Hepatic veno-occlusive disease related to tacrolimus after pancreas transplantation. J Chin Med Assoc 76: 358-360, 2013.

12. Shulman HM, Gown AM and Nugent DJ: Hepatic veno-occlusive disease after bone marrow transplantation. Immunohistochemical identification of the material within occluded central venules. Am J Pathol 127: 549-558, 1987.

13. Cogger VC, McNerney GP and Nyunt T: Three-dimensional structured illumination microscopy of liver sinusoidal endothelial cell fenestrations. J Struct Biol 171: 382-388, 2010.

14. Mak KM and Lieber CS: Alterations in endothelial fenestrations in liver sinusoids of baboons fed alcohol: a scanning electron microscopic study. Hepatology 4: 386-391, 1984.

15. Yamamoto M, Sumiyoshi H, Nakagami K and Tahara E: Distribution of collagen types I, III, and V in fibrotic and neoplastic human liver. Acta Pathol Jpn 34: 77-86, 1984.

16. Jung SM, Takemura Y, Imamura Y, et al: Collagen-type specificity of glycoprotein VI as a determinant of platelet adhesion. Platelets 19: 32-42, 2008.

17. Brech A, Kjeken R, Synnes M, et al: Endocytosed ricin and asialoorosomucoid follow different intracellular pathways in hepatocytes. Biochim Biophys Acta 1373: 195-208, 1998.

18. Steirer LM, Park EI, Townsend RR, et al: The asialoglycoprotein receptor regulates levels of plasma glycoproteins terminating with sialic acid alpha2,6-galactose. J Biol Chem 284: 3777-3783, 2009.

19. Sørensen AL, Rumjantseva V, Nayeb-Hashemi S, et al: Role of sialic acid for platelet life span: exposure of beta-galactose results in the rapid clearance of platelets from the circulation by asialoglycoprotein receptor-expressing liver macrophages and hepatocytes. Blood 114: 1645-1654, 2009.

20. Golebiewska EM and Poole AW: Platelet secretion: From haemostasis to wound healing and beyond. Blood Rev: Oct 31, 2014 (Epub ahead of print), doi: 10.1016/j.blre.2014.10.003.

21. Mohammad SF, Anderson WH, Smith JB, et al: Effects of heparin on platelet aggregation and release and thromboxane A2 production. Am J Pathol 104:132-141, 1981.

22. Anitua E, Andia I, Ardanza B, et al: Autologous platelets as a source of proteins for healing and tissue regeneration. Thromb Haemost 91:4-15, 2004.

23. Ruan Z, Shibamoto T, Shimo T, et al: Effects of platelet-activating factor and thromboxane A2 on isolated perfused guinea pig liver. Prostaglandins Other Lipid Mediat 73: 73-85, 2004.

24. Parenti A, Brogelli L, Filippi S, et al: Effect of hypoxia and endothelial loss on vascular smooth muscle cell responsiveness to VEGF-A: role of fit-1/VEGF-receptor-1. Cardiovasc Res 55: 201-212, 2002.

25. Ribero D, Wang H, Donadon M, et al: Bevacizumab improves pathologic response and protects against hepatic injury in patients treated with oxaliplatin-based chemotherapy for colorectal liver metastases. Cancer 110: 2761-2767, 2007.

26. Mars WM, Zarnegar R and Michalopoulos GK: Activation of hepatocyte growth factor by the plasminogen activators uPA and tPA. Am J Pathol 143: 949-958, 1993.

27. Watanabe K, Togo S, Takahashi T, et al: PAI-1 plays an important role in liver failure after excessive hepatectomy in the rat. J Surg Res 143: 13-19, 2007

28. Ueda S, Yamanoi A, Hishikawa Y, et al: Transforming growth factor-betal released from the spleen exerts a growth inhibitory effect on liver regeneration in rats. Lab Invest 83: 1595-1603, 2003.

29. Watanabe T, Tajima H, Hironori H, et al: Sodium valproate blocks the transforming growth factor (TGF)- $\beta 1$ autocrine loop and attenuates the TGF- $\beta 1$-induced collagen synthesis in a human hepatic stellate cell line. Int J Mol Med 28: 919-925, 2011. 
30. Yamada N, Urahashi $\mathrm{T}$, Ihara $\mathrm{Y}$, et al: Veno-occlusive disease/sinusoidal obstruction syndrome associated with potential antibody-mediated rejection after pediatric living donor liver transplantation: a case report. Transplant Proc 44: 810-813, 2012.

31. Zhang L, Wang Y and Huang H: Defibrotide for the prevention of hepatic veno-occlusive disease after hematopoietic stem cell transplantation: a systematic review. Clin Transplant 26: 511-519, 2012.
32. Iba T, Kidokoro A, Fukunaga M, et al: Comparison of the protective effects of type III phosphodiesterase (PDE3) inhibitor (cilostazol) and acetylsalicylic acid on intestinal microcirculation after ischemia reperfusion injury in mice. Shock 26: 522-526, 2006.

33. Wang S, Yan C, Xu H, Zhao X and Han Y: Suppression of encephalitogenic T-cell responses by cilostazol is associated with upregulation of regulatory T cells. Neuroreport 21: 629-635, 2010 . 